\title{
When in Rome... Be Yourself: A Perspective on Dealing with Cultural Dissimilarities in Ethnography
}

\author{
Apala Lahiri Chavan ${ }^{1}$ and Rahul Ajmera ${ }^{2}$ \\ ${ }^{1}$ Vice President - Asia \\ Human Factors International \\ Chemtex House, $4^{\text {th }}$ Floor \\ Hiranandani Gardens, Powai \\ Mumbai 400072 \\ apala@humanfactors.com \\ ${ }^{2}$ Project Manager \\ Human Factors International India Pvt. Ltd. \\ 310/6, H.R. Complex, 2nd Floor, \\ Koramangala, 5th Block, \\ Bangalore - 500095 \\ Tel.: +91 $8041507221 / 2 / 3$ \\ Fax: +91 8041507220 \\ rahul@humanfactors.com
}

\begin{abstract}
With the 'flattening' of the world, increasingly, our design research teams are called upon to execute projects in cultures that are foreign to them. Design research involves deep dive ethnography that needs to be carried out in a relatively short span of time. It is in these design ethnography studies that we have realized the impact of cultural difference between the researchers and the researched. This paper attempts to discuss our findings on the subject.
\end{abstract}

\section{Introduction}

Human Factors International Inc. (HFI) is a 240 people, \$20 million consulting practice working in the area of user centred design, with a mission to improve the interactions that people have with computers and other digital systems. HFI offers end to end solutions for Web/Intranet and Internet-based applications, Software Applications, IVR Systems, Handheld Devices, Telemetric, Public Service Networks, Medical and Automation Equipments and help make our clients' existing offerings more user centric, optimized and efficient. In the wake of the recent interest in research and development for business innovations for the emerging markets and products \& services for new markets, we have established ethnography and design research as a service area along with the existing areas of HFI's activities \& services. HFI's interest in this area is reflected through successful collaborations with research initiatives launched by global corporate as well as academic institutions, e.g. HP Labs, Nokia Research, NCR, Media Lab Asia, Intel etc.

\section{Role of Ethnography in Innovation}

As technology permeates each and every aspect of our lives, we are constantly faced with situations where there are mismatches between its role and our lives. It is now 
known even to technologists that the power of technology can only be gauged by its social relevance and acceptability. Understanding how users experience technologies necessitates a concern with social and cultural meaning; what does the product mean to the user; what does it mean in the context of particular cultures, what does it mean in terms of its broad social and global environment? [1]. Socio cultural relevance is not limited to technological products or services but it is given a lot of importance as development costs associated with development of technologies are typically very high and the development cycles very long. These high stakes make it imperative that a reliable requirement capture method be implemented.

\subsection{Ethnography: Introduction}

Ethnography, is a traditional method that belongs to the fields of sociology and cultural anthropology. It involves the study of people performing activities and interacting in complex social settings in order to obtain a qualitative understanding of these interactions [2]. Classic ethnographic analysis is typically based on extensive interviews, observations and field studies that consume months or years. This immersion in the world of study participants permits the investigator to create a detailed, fine grained picture of a culture. The time intensive nature of these conventional ethnographic studies however rarely ever seem to fit corporate cultures and their technology development time lines. In addition, the discursive, qualitative descriptions necessary to present this rich picture does not often align well with formal system specifications [3]. Nonetheless the benefits of examining field situated user activity remains inviting [4].

In response, classic ethnographic studies have been modified to accommodate commercial and educational circumstances, to allow practitioners and students to gain insights more quickly and more directly from potential end users.

Our studies typically include depth interviews, cultural probes, contextual inquiry, observational studies etc. The techniques and methods we employ are modified versions of methods borrowed from traditional ethnography. We at HFI refer to our overall innovation process as "Contextual Innovation". While we effectively employ ethnography based methods it is also important for us to realize that there are trade offs with this resource effective industry driven version of ethnography. Though the scope of this paper does not permit discussion of the short falls but some of the issues would be raised as they are directly related to the focus of the paper, that is, understanding the effect of cultural differences on ethnography.

\section{Cultural Differences}

As we move from project to project we have conducted studies in a variety of different socio-cultural contexts varying sometimes from a household in New York to one in rural India. In a world that is rapidly globalizing, the instances of this are only going to increase for user researchers around the world. In many cases our researchers have had to conduct their studies in cultures fairly alien to them. It is during these studies that we have come to realize the importance and the impact of these cultural differences. In this paper we will try to discuss areas where we have realized these differences play a major role and how they can be addressed. 


\subsection{Addressing Cultural Differences: Traditional Ethnography vs Design Ethnography}

One of the important differences between our kind of research vs traditional ethnography is the time the researchers spend on the field. Typically our researchers segment the study into focused visits during which they attempt to unearth the most relevant aspects of the contexts. These visits are not long enough for the researcher and the researched to be completely at ease. 'There is an assumption that as the researcher becomes a more familiar presence, participants are less likely to behave uncharacteristically".

While we would like to believe that our attempts at making the participants comfortable, work, but in doing so the dynamics still cannot always be compared to that in traditional ethnography. This implies that when researchers from foreign cultures enter an unknown context for short periods(as is mostly the case with design ethnography) the effect of this cultural difference may impact the study adversely .

One clear impact on the study in such cases is that researchers themselves stand out in the context. This point is of great interest because there are a lot of researchers who stress on trying to "merge into the researched contexts". Their prime reason of advocating such an approach to handle "the public glare" is to make the participants comfortable. We have in some cases tried to follow this approach but have realized that, sometimes, in spite of our best efforts we could not "merge in" and trying to awkwardly deal with this inability to immerse ourselves in the context of our participants only made us feel more uncomfortable. In our opinion, rather than struggle with an impossible immersion, the better approach is to embrace the 'nonimmersion' and to actually use the 'foreignness' of the researcher to our advantage.

After all, in ethnography, the researcher deliberately constitutes himself as the "other" in embarking on the enterprise of fieldwork. Having become the 'other', the researcher, in classical ethnography, tries to make sense of the 'lived' experience of the 'people' he is trying to understand. The 'key informant' from amongst the 'natives' plays a critical role in helping the researcher understand this 'lived experience.

When we say that using the 'foreignness' is often more helpful rather than trying to immerse ourselves (as researcher) into the 'lived' experience, one primary reason is that we have, in this abridged form of ethnography, 6 days available rather than 6 months. Hence, while we very much constitute ourselves as the 'other', what we do differently is that often we do not go in for immersion in the context of our participants.

And this leads us to the much debated concepts of 'emic' and 'etic' knowledge.

\section{Neutrality vs Immersion}

'A researcher who works with emic knowledge, will look into - and generally accept unproblematically - the rules, terms, reference points and logic of the person she is studying. Part of what she will convey to readers of her research is this internal system of logic of the group or person, and her conclusions will derive from that. The 
analogy to a phoneme is clear - in linguistics it denotes a meaningful unit of sound specific to a particular language.

An etic researcher will ask her informant questions based on her own perspective and concerns, which are often seen to be 'scientific', or 'universal'. Phonetics, which discusses sounds qua units of sound, rather than sounds in context, is a fitting theoretical analogy. The researcher will present an interpretation of her data that draws conclusions using external categories, valuations, and judgments. In the social sciences today claims of scientific methods, universalism, and neutrality are heavily contested.

A simple way of thinking about the distinction is this: an emic researcher will 'go native' to some extent, behaving, speaking, eating, and thinking like her subjects of study. An etic researcher will stay on the edges, assessing them on her own terms.'[6].

\section{Conclusion - Stranger in a Strange Land!}

When working with abridged ethnographic methods, as in design research, the 'etic' approach often elicits more open and honest responses from the participants. The feeling that participants get, of, 'oh this chap is a foreigner and therefore its ok that he is asking such strange/stupid questions' makes it much easier for us to ask questions that would normally be thought of as 'awkward' or even a 'strict no-no' and equally easy for the participants to answer what would otherwise be considered embarrassing or very personal questions.

Moreover, the process we follow when working on contextual innovation projects places considerable emphasis on understanding the clients ecosystem. Thus, while we might be foreign to the context, this emphasis gives us a framework to probe from the 'outside' if necessary and be familiar with the participants' "perspective". It is this understanding of the perspective that is taken into account in our etic "Point of view".

As Pawan Verma says [7], 'societies reveal how they actually think and behave in the smallest things. Behavioural patterns have to be discovered not in the considered stance before an observer, but in the insignificant reflex preceding or following it'.

Being an 'outsider' trained in design ethnography, it often becomes easier to assume that there IS a 'considered stance' and therefore be on the lookout for the 'insignificant reflex'.

\section{References}

1. Bell, G., Blythe, M., Gaver, B., Sengers, P., Wright, P.: Designing Culturally situated Technologies for the home. CHI 2003 (2003)

2. McCleverty, A.: Ethnography. Computer science 681: research methodologies (1997)

3. Hughes, J., King, V., Rodden, T., Anderson, H.: The role of ethnography in interactive systems design. Interactions (1995)

4. Millen, D.: Rapid ethnography: Time deepening strategies for HCI

5. Anderson, R.: Representations and requirements: The value of ethnography in System design. HCI 1992 (1992)

6. http://www.articleworld.org/index.php/Emic_and_etic

7. Varma, P.: Being Indian. Penguin Books India, New Delhi (2004) 